International Journal of Algebra, Vol. 1, 2007, no. 6, 269 - 281

\title{
A New Bartholdi Zeta Function of a Graph
}

\author{
Iwao Sato ${ }^{1}$ \\ Oyama National College of Technology \\ Oyama, Tochigi 323-0806, Japan \\ isato@oyama-ct.ac.jp
}

\begin{abstract}
We define a new type of the Bartholdi zeta function of a graph $G$, and give a determinant expression of it. Furthermore, we define a new type of the Bartholdi $L$-function of $G$, and present a determinant expression for a new type of the Bartholdi $L$-function of $G$. As a corollary, we show that a new type of the Bartholdi zeta function of a regular covering of $G$ is a product of new Batholdi $L$-functions of $G$.
\end{abstract}

Mathematics Subject Classification: 05C50, 05C25, 05C10, 15A15

Keywords: zeta function, graph covering, $L$-function

\section{Introduction}

Graphs treated here are finite. Let $G=(V(G), E(G))$ be a connected graph (possibly multiple edges and loops) with the set $V(G)$ of vertices and the set $E(G)$ of unoriented edges $u v$ joining two vertices $u$ and $v$. For $u v \in E(G)$, an $\operatorname{arc}(u, v)$ is the oriented edge from $u$ to $v$. Set $D(G)=\{(u, v),(v, u) \mid u v \in$ $E(G)\}$. For $e=(u, v) \in D(G)$, set $u=o(e)$ and $v=t(e)$. Furthermore, let $e^{-1}=(v, u)$ be the inverse of $e=(u, v)$.

A path $P$ of length $n$ in $G$ is a sequence $P=\left(e_{1}, \ldots, e_{n}\right)$ of $n$ arcs such that $e_{i} \in D(G), t\left(e_{i}\right)=o\left(e_{i+1}\right)(1 \leq i \leq n-1)$, where indices are treated $\bmod n$. Set $|P|=n, o(P)=o\left(e_{1}\right)$ and $t(P)=t\left(e_{n}\right)$. Also, $P$ is called an $(o(P), t(P))$-path. We say that a path $P=\left(e_{1}, \ldots, e_{n}\right)$ has a backtracking if $e_{i+1}^{-1}=e_{i}$ for some $i(1 \leq i \leq n-1)$. A $(v, w)$-path is called a $v$-cycle (or $v$-closed path) if $v=w$. The inverse cycle of a cycle $C=\left(e_{1}, \ldots, e_{n}\right)$ is the cycle $C^{-1}=\left(e_{n}^{-1}, \ldots, e_{1}^{-1}\right)$.

We introduce an equivalence relation between cycles. Two cycles $C_{1}=$ $\left(e_{1}, \ldots, e_{m}\right)$ and $C_{2}=\left(f_{1}, \ldots, f_{m}\right)$ are called equivalent if there exists $k$ such that $f_{j}=e_{j+k}$ for all $j$. The inverse cycle of $C$ is in general not equivalent to $C$.

\footnotetext{
${ }^{1}$ Supported by Grant-in-Aid for Science Research (C)
} 
Let $[C]$ be the equivalence class which contains a cycle $C$. Let $B^{r}$ be the cycle obtained by going $r$ times around a cycle $B$. Such a cycle is called a power of $B$. A cycle $C$ is reduced if both $C$ and $C^{2}$ have no backtracking. Furthermore, a cycle $C$ is prime if it is not a power of a strictly smaller cycle. Note that each equivalence class of prime, reduced cycles of a graph $G$ corresponds to a unique conjugacy class of the fundamental group $\pi_{1}(G, v)$ of $G$ at a vertex $v$ of $G$.

Let $G$ be a connected graph. For each $u, v \in V(G)$, let $[u, v]$ be the set of all $(u, v)$-paths in $G$. We say that a path $P=\left(e_{1}, \cdots, e_{n}\right)$ has a bump at $t\left(e_{i}\right)$ if $e_{i+1}=e_{i}^{-1}(1 \leq i \leq n)$. The bump count $b c(P)$ of a path $P$ is the number of bumps in $P$. Furthermore, the cyclic bump count $\operatorname{cbc}(\pi)$ of a cycle $\pi=\left(\pi_{1}, \ldots, \pi_{n}\right)$ is

$$
\operatorname{cbc}(\pi)=\left|\left\{i=1, \ldots, n \mid \pi_{i}=\pi_{i+1}^{-1}\right\}\right|,
$$

where $\pi_{n+1}=\pi_{1}$. Then the Bartholdi zeta function of $G$ is defined to be a function of $u, t \in \mathbf{C}$ with $|u|,|t|$ sufficiently small, by

$$
\zeta_{G}(u, t)=\zeta(G, u, t)=\prod_{[C]}\left(1-u^{c b c(C)} t^{|C|}\right)^{-1}
$$

where $[C]$ runs over all equivalence classes of prime cycles of $G$ (see [1]).

Bartholdi [1] gave a determinant expression of the Bartholdi zeta function of a graph.

Theorem 1 (Bartholdi) Let $G$ be a connected graph with $n$ vertices and $m$ unoriented edges. Then the reciprocal of the Bartholdi zeta function of $G$ is given by

$$
\zeta(G, u, t)^{-1}=\left(1-(1-u)^{2} t^{2}\right)^{m-n} \operatorname{det}\left(\mathbf{I}-t \mathbf{A}(G)+(1-u)(\mathbf{D}-(1-u) \mathbf{I}) t^{2}\right),
$$

where $\mathbf{A}(G)$ is the adjacency matrix of $G$, and $\mathbf{D}=\left(d_{i j}\right)$ is the diagonal matrix with $d_{i i}=\operatorname{deg} v_{i}$ where $V(G)=\left\{v_{1}, \ldots, v_{n}\right\}$.

If $u=0$, then the Bartholdi zeta function of $G$ is the (Ihara) zeta function of a graph $G$ :

$$
\mathbf{Z}(G, u)=\mathbf{Z}_{G}(u)=\prod_{[C]}\left(1-u^{|C|}\right)^{-1},
$$

where $[C]$ runs over all equivalence classes of prime, reduced cycles of $G$ (see $[6])$.

Zeta functions of graphs started from zeta functions of regular graphs by Ihara [6]. In [6], he showed that their reciprocals are explicit polynomials. A zeta function of a regular graph $G$ associated with a unitary representation of 
the fundamental group of $G$ was developed by Sunada $[12,13]$. Hashimoto [5] treated multivariable zeta functions of bipartite graphs. Bass [2] generalized Ihara's result on the zeta function of a regular graph to an irregular graph, and showed that its reciprocal is again a polynomial:

$$
\mathbf{Z}(G, u)^{-1}=\left(1-u^{2}\right)^{r-1} \operatorname{det}\left(\mathbf{I}-u \mathbf{A}(G)+u^{2}(\mathbf{D}-\mathbf{I})\right) .
$$

Stark and Terras [12] gave an elementary proof of Bass' Theorem, and discussed three different zeta functions of any graph. Furthermore, various proofs of Bass' Theorem were given by Foata and Zeilberger [3], Kotani and Sunada [7].

Sato [9] defined a new zeta function of a graph by using not an infinite product but a determinant.

Let $G$ be a connected graph and $V(G)=\left\{v_{1}, \cdots, v_{n}\right\}$. Then we consider an $n \times n$ matrix $\mathbf{W}=\left(w_{i j}\right)_{1 \leq i, j \leq n}$ with $i j$ entry the complex variable $w_{i j}$ if $\left(v_{i}, v_{j}\right) \in D(G)$, and $w_{i j}=0$ otherwise. The matrix $\mathbf{W}=\mathbf{W}(G)$ is called the weighted matrix of $G$. For each path $P=\left(v_{i_{1}}, \cdots, v_{i_{r}}\right)$ of $G$, the norm $w(P)$ of $P$ is defined as follows: $w(P)=w_{i_{1} i_{2}} w_{i_{2} i_{3}} \cdots w_{i_{r-1} i_{r}}$. Furthermore, let $w\left(v_{i}, v_{j}\right)=w_{i j}, v_{i}, v_{j} \in V(G)$ and $w(e)=w_{i j}, e=\left(v_{i}, v_{j}\right) \in D(G)$.

Let $G$ be a connected graph with $n$ vertices and $m$ unoriented edges, and $\mathbf{W}=\mathbf{W}(G)$ a weighted matrix of $G$. Two $2 m \times 2 m$ matrices $\mathbf{B}=\mathbf{B}(G)=$ $\left(\mathbf{B}_{e, f}\right)_{e, f \in D(G)}$ and $\mathbf{J}_{0}=\mathbf{J}_{0}(G)=\left(\mathbf{J}_{e, f}\right)_{e, f \in D(G)}$ are defined as follows:

$$
\mathbf{B}_{e, f}=\left\{\begin{array}{ll}
w(f) & \text { if } t(e)=o(f), \\
0 & \text { otherwise }
\end{array}, \mathbf{J}_{e, f}= \begin{cases}1 & \text { if } f=e^{-1} \\
0 & \text { otherwise }\end{cases}\right.
$$

Then the zeta function of $G$ is defined by

$$
\mathbf{Z}_{1}(G, w, u)=\operatorname{det}\left(\mathbf{I}_{n}-u\left(\mathbf{B}-\mathbf{J}_{0}\right)\right)^{-1}
$$

If $w(e)=1$ for any $e \in D(G)$, then the zeta function of $G$ is the Ihara zeta function of $G$.

Let $\mathbf{M}_{1} \oplus \cdots \oplus \mathbf{M}_{s}$ be the block diagonal sum of square matrices $\mathbf{M}_{1}, \cdots, \mathbf{M}_{s}$.

Theorem 2 Let $G$ be a connected graph, and let $\mathbf{W}=\mathbf{W}(G)$ be a weighted matrix of $G$. Then the reciprocal of the zeta function of $G$ is given by

$$
\mathbf{Z}_{1}(G, w, u)^{-1}=\left(1-u^{2}\right)^{m-n} \operatorname{det}\left(\mathbf{I}_{n}-u \mathbf{W}(G)+u^{2}\left(\mathbf{D}-\mathbf{I}_{n}\right)\right)
$$

where $n=|V(G)|, m=|E(G)|$ and $\mathbf{D}=\left(d_{i j}\right)$ is the diagonal matrix with $d_{i i}=\sum_{o(e)=v_{i}} w(e), V(G)=\left\{v_{1}, \cdots, v_{n}\right\}$.

In Section 2, we define a new type of the Bartholdi zeta function of a graph $G$, and give a determinant expression of it. In Section 3, we give a decompostion formula of a new type of the Bartholdi zeta function of a regular 
covering of $G$. In Section 4, we define a new type of the Bartholdi $L$-function of $G$, and present a determinant expression for a new type of the Bartholdi $L$-function of $G$. Furthermore, we show that a new type of the Bartholdi zeta function of a regular covering of $G$ is a product of new Bartholdi $L$-functions of $G$.

A general theory of the representation of groups and graph coverings, the reader is referred to [10] and [4], respectively.

\section{A New Bartholdi zeta functions of graphs}

We consider a new zeta function of a graph under Theorems 1,2, and define a new zeta function of a graph by using not an infinite product but a determinant.

Let $G$ be a connected graph and $V(G)=\left\{v_{1}, \cdots, v_{n}\right\}$. Then we consider an $n \times n$ matrix $\mathbf{W}=\left(w_{i j}\right)_{1 \leq i, j \leq n}$ with $i j$ entry the complex variable $w_{i j}$ if $\left(v_{i}, v_{j}\right) \in D(G)$, and $w_{i j}=0$ otherwise. The matrix $\mathbf{W}=\mathbf{W}(G)$ is called the weighted matrix of $G$. For each path $P=\left(v_{i_{1}}, \cdots, v_{i_{r}}\right)$ of $G$, the norm $w(P)$ of $P$ is defined as follows: $w(P)=w_{i_{1} i_{2}} w_{i_{2} i_{3}} \cdots w_{i_{r-1} i_{r}}$. Furthermore, let $w\left(v_{i}, v_{j}\right)=w_{i j}, v_{i}, v_{j} \in V(G)$ and $w(e)=w_{i j}, e=\left(v_{i}, v_{j}\right) \in D(G)$.

Let $G$ be a connected graph with $n$ vertices and $m$ unoriented edges, and $\mathbf{W}=\mathbf{W}(G)$ a weighted matrix of $G$. Two $2 m \times 2 m$ matrices $\mathbf{B}=\mathbf{B}(G)=$ $\left(\mathbf{B}_{e, f}\right)_{e, f \in D(G)}$ and $\mathbf{J}_{0}=\mathbf{J}_{0}(G)=\left(\mathbf{J}_{e, f}\right)_{e, f \in D(G)}$ are defined as follows:

$$
\mathbf{B}_{e, f}=\left\{\begin{array}{ll}
w(f) & \text { if } t(e)=o(f), \\
0 & \text { otherwise }
\end{array}, \mathbf{J}_{e, f}=\left\{\begin{array}{ll}
1 & \text { if } f=e^{-1} \\
0 & \text { otherwise }
\end{array},\right.\right.
$$

Then the zeta function of $G$ is defined by

$$
\zeta_{1}(G, w, u, t)=\operatorname{det}\left(\mathbf{I}_{n}-t\left(\mathbf{B}-(1-u) \mathbf{J}_{0}\right)\right)^{-1} .
$$

If $w(e)=1$ for any $e \in D(G)$, then the zeta function of $G$ is the Bartholdi zeta function of $G$ (see [1]). In the case of $u=0$, then the zeta function of $G$ is the new zeta function of $G$ (see [9]).

Let $\mathbf{M}_{1} \oplus \cdots \oplus \mathbf{M}_{s}$ be the block diagonal sum of square matrices $\mathbf{M}_{1}, \cdots, \mathbf{M}_{s}$.

Theorem 3 Let $G$ be a connected graph, and let $\mathbf{W}=\mathbf{W}(G)$ be a weighted matrix of $G$. Then the reciprocal of the zeta function of $G$ is given by

$\zeta_{1}(G, w, u, t)^{-1}=\left(1-(1-u)^{2} t^{2}\right)^{m-n} \operatorname{det}\left(\mathbf{I}_{n}-t \mathbf{W}(G)+(1-u) t^{2}\left(\mathbf{D}-(1-u) \mathbf{I}_{n}\right)\right)$,

where $n=|V(G)|, m=|E(G)|$ and $\mathbf{D}=\left(d_{i j}\right)$ is the diagonal matrix with $d_{i i}=\sum_{o(e)=v_{i}} w(e), V(G)=\left\{v_{1}, \cdots, v_{n}\right\}$.

Proof. Similar to the proof of Theorem 6.

If $u=0$, then Theorem 3 implies Theorem 2 . 


\section{Zeta functions of regular coverings of graphs}

Let $G$ be a connected graph, and let $N(v)=\{w \in V(G) \mid(v, w) \in D(G)\}$ denote the neighbourhood of a vertex $v$ in $G$. A graph $H$ is called a covering of $G$ with projection $\pi: H \longrightarrow G$ if there is a surjection $\pi: V(H) \longrightarrow V(G)$ such that $\left.\pi\right|_{N\left(v^{\prime}\right)}: N\left(v^{\prime}\right) \longrightarrow N(v)$ is a bijection for all vertices $v \in V(G)$ and $v^{\prime} \in \pi^{-1}(v)$. When a finite group $\Pi$ acts on a graph $G$, the quotient graph $G / \Pi$ is a graph whose vertices are the $\Pi$-orbits on $V(G)$, with two vertices adjacent in $G / \Pi$ if and only if some two of their representatives are adjacent in $G$. A covering $\pi: H \longrightarrow G$ is said to be regular if there is a subgroup $B$ of the automorphism group $A$ ut $H$ of $H$ acting freely on $H$ such that the quotient graph $H / B$ is isomorphic to $G$.

Let $G$ be a graph and $\Gamma$ a finite group. Then a mapping $\alpha: D(G) \longrightarrow \Gamma$ is called an ordinary voltage assignment if $\alpha(v, u)=\alpha(u, v)^{-1}$ for each $(u, v) \in$ $D(G)$. The pair $(G, \alpha)$ is called an ordinary voltage graph. The derived graph $G^{\alpha}$ of the ordinary voltage graph $(G, \alpha)$ is defined as follows: $V\left(G^{\alpha}\right)=V(G) \times$ $\Gamma$ and $((u, h),(v, k)) \in D\left(G^{\alpha}\right)$ if and only if $(u, v) \in D(G)$ and $k=h \alpha(u, v)$. The natural projection $\pi: G^{\alpha} \longrightarrow G$ is defined by $\pi(u, h)=u$. The graph $G^{\alpha}$ is called a derived graph covering of $G$ with voltages in $\Gamma$ or a $\Gamma$-covering of $G$. The natural projection $\pi$ commutes with the right multiplication action of the $\alpha(e), e \in D(G)$ and the left action of $\Gamma$ on the fibers: $g(u, h)=(u, g h), g \in \Gamma$, which is free and transitive. Thus, the $\Gamma$-covering $G^{\alpha}$ is a $|\Gamma|$-fold regular covering of $G$ with covering transformation group $\Gamma$. Futhermore, every regular covering of a graph $G$ is a $\Gamma$-covering of $G$ for some group $\Gamma$ (see [4]).

Let $G$ be a connected graph, $\Gamma$ a finite group and $\alpha: D(G) \longrightarrow \Gamma$ an ordinary voltage assignment. In the $\Gamma$-covering $G^{\alpha}$, set $v_{g}=(v, g)$ and $e_{g}=$ $(e, g)$, where $v \in V(G), e \in D(G), g \in \Gamma$. For $e=(u, v) \in D(G)$, the arc $e_{g}$ emanates from $u_{g}$ and terminates at $v_{g \alpha(e)}$. Note that $e_{g}^{-1}=\left(e^{-1}\right)_{g \alpha(e)}$.

Let $\mathbf{W}=\mathbf{W}(G)$ be a weighted matrix of $G$. Then we define the weighted matrix $\tilde{\mathbf{W}}=\mathbf{W}\left(G^{\alpha}\right)=\left(\tilde{w}\left(u_{g}, v_{h}\right)\right)$ of $G^{\alpha}$ derived from $\mathbf{W}$ as follows:

$$
\tilde{w}\left(u_{g}, v_{h}\right):= \begin{cases}w(u, v) & \text { if }(u, v) \in D(G) \text { and } h=g \alpha(u, v), \\ 0 & \text { otherwise. }\end{cases}
$$

For $g \in \Gamma$, let the matrix $\mathbf{W}_{g}=\left(w_{u v}^{(g)}\right)$ be defined by

$$
w_{u v}^{(g)}:= \begin{cases}w(u, v) & \text { if } \alpha(u, v)=g \text { and }(u, v) \in D(G), \\ 0 & \text { otherwise. }\end{cases}
$$

If $\mathbf{M}_{1}=\mathbf{M}_{2}=\cdots=\mathbf{M}_{s}=\mathbf{M}$, then we write $s \circ \mathbf{M}=\mathbf{M}_{1} \oplus \cdots \oplus \mathbf{M}_{s}$. The Kronecker product $\mathbf{A} \otimes \mathbf{B}$ of matrices $\mathbf{A}$ and $\mathbf{B}$ is considered as the matrix $\mathbf{A}$ having the element $a_{i j}$ replaced by the matrix $a_{i j} \mathbf{B}$.

Theorem 4 Let $G$ be a connected graph with $\nu$ vertices and $\epsilon$ unoriented edges, $\mathbf{W}=\mathbf{W}(G)$ a weighted matrix of $G, \Gamma$ a finite group and $\alpha: D(G) \longrightarrow \Gamma$ 
an ordinary voltage assignment. Furthermore, let $\rho_{1}=1, \rho_{2}, \cdots, \rho_{k}$ be the irreducible representations of $\Gamma$, and $f_{i}$ the degree of $\rho_{i}$ for each $i$, where $f_{1}=1$. Suppose that the $\Gamma$-covering $G^{\alpha}$ of $G$ is connected. Then the reciprocal of the zeta function of $G^{\alpha}$ is

$$
\begin{gathered}
\zeta_{1}\left(G^{\alpha}, \tilde{w}, u, t\right)^{-1}=\zeta_{1}(G, w, u, t)^{-1} \\
\cdot \prod_{i=2}^{k}\left\{( 1 - ( 1 - u ) ^ { 2 } t ^ { 2 } ) ^ { ( \epsilon - \nu ) f _ { i } } \operatorname { d e t } \left(\mathbf{I}_{\nu f_{i}}-t \sum_{h \in \Gamma} \rho_{i}(h) \bigotimes \mathbf{W}_{h}\right.\right. \\
\left.\left.+(1-u) t^{2}\left(\mathbf{I}_{f_{i}} \bigotimes\left(\mathbf{D}-(1-u) \mathbf{I}_{\nu}\right)\right)\right)\right\}^{f_{i}}
\end{gathered}
$$

Proof. Let $V(G)=\left\{v_{1}, \cdots, v_{\nu}\right\}$ and $\Gamma=\left\{1=g_{1}, g_{2}, \cdots, g_{m}\right\}$. Arrange arcs of $G^{\alpha}$ in $m$ blocks: $\left(v_{1}, 1\right), \cdots,\left(v_{\nu}, 1\right) ;\left(v_{1}, g_{2}\right), \cdots,\left(v_{\nu}, g_{2}\right) ; \cdots ;\left(v_{1}, g_{m}\right), \cdots$ ,$\left(v_{\nu}, g_{m}\right)$. We consider the adjacency matrix $\mathbf{W}\left(G^{\alpha}\right)$ under this order. For $h \in \Gamma$, the matrix $\mathbf{P}_{h}=\left(p_{i j}^{(h)}\right)$ is defined as follows:

$$
p_{i j}^{(h)}= \begin{cases}1 & \text { if } g_{i} h=g_{j} \\ 0 & \text { otherwise }\end{cases}
$$

Suppose that $p_{i j}^{(h)}=1$, i.e., $g_{j}=g_{i} h$. Then $\left(\left(u, g_{i}\right),\left(v, g_{j}\right)\right) \in D\left(G^{\alpha}\right)$ if and only if $(u, v) \in D(G)$ and $g_{j}=g_{i} \alpha(u, v)$, i.e., $\alpha(u, v)=g_{i}^{-1} g_{j}=g_{i}^{-1} g_{i} h=h$. Thus we have

$$
\mathbf{W}\left(G^{\alpha}\right)=\sum_{h \in \Gamma} \mathbf{P}_{h} \bigotimes \mathbf{W}_{h}
$$

Let $\rho$ be the right regular representation of $\Gamma$. Furthermore, let $\rho_{1}=$ $1, \rho_{2}, \cdots, \rho_{k}$ be the irreducible representations of $\Gamma$, and $f_{i}$ the degree of $\rho_{i}$ for each $i$, where $f_{1}=1$. Then we have $\rho(h)=\mathbf{P}_{h}$ for $h \in \Gamma$. Furthermore, there exists a nonsingular matrix $\mathbf{P}$ such that $\mathbf{P}^{-1} \rho(h) \mathbf{P}=(1) \oplus f_{2} \circ \rho_{2}(h) \oplus \cdots \oplus$ $f_{k} \circ \rho_{k}(h)$ for each $h \in \Gamma$ (see [10]). Putting $\mathbf{F}=\left(\mathbf{P}^{-1} \bigotimes \mathbf{I}_{\nu}\right) \mathbf{W}\left(G^{\alpha}\right)\left(\mathbf{P} \bigotimes \mathbf{I}_{\nu}\right)$, we have

$$
\mathbf{F}=\sum_{h \in \Gamma}\left\{(1) \oplus f_{2} \circ \rho_{2}(h) \oplus \cdots \oplus f_{k} \circ \rho_{k}(h)\right\} \bigotimes \mathbf{W}_{h}
$$

Note that $\mathbf{W}(G)=\sum_{h \in \Gamma} \mathbf{W}_{h}$ and $1+f_{2}^{2}+\cdots+f_{k}^{2}=m$. Therefore it follows that

$$
\begin{aligned}
& \zeta_{1}\left(G^{\alpha}, \tilde{w}, u, t\right)^{-1}=\left(1-(1-u)^{2} t^{2}\right)^{(\epsilon-\nu) m} \operatorname{det}\left(\mathbf{I}_{\nu m}-t \mathbf{W}\left(G^{\alpha}\right)+(1-u)\left(\mathbf{I}_{m} \otimes\left(\mathbf{D}-(1-u) \mathbf{I}_{\nu}\right)\right) t^{2}\right) \\
& =\left(1-(1-u)^{2} t^{2}\right)^{\epsilon-\nu} \operatorname{det}\left(\mathbf{I}_{\nu}-t \mathbf{W}(G)+(1-u)\left(\mathbf{D}-(1-u) \mathbf{I}_{\nu}\right) t^{2}\right)
\end{aligned}
$$


We give another decomposition formula for the zeta function of a regular covering $G^{\alpha}$ of a graph $G$.

Let $G$ be a connected graph, $\Gamma$ a finite group and $\alpha: D(G) \longrightarrow \Gamma$ an ordinary voltage assignment. Let $w: D(G) \longrightarrow \mathbf{C}$ be a weight of $G$. Then two matrices $\tilde{\mathbf{B}}=\mathbf{B}\left(G^{\alpha}\right)=\left(\tilde{b}\left(e_{g}, f_{h}\right)\right)$ and $\tilde{\mathbf{J}}_{0}=\mathbf{J}_{0}\left(G^{\alpha}\right)=\left(\tilde{c}\left(e_{g}, f_{h}\right)\right)$ of $G^{\alpha}$ are given by

$$
\tilde{b}\left(e_{g}, f_{h}\right):= \begin{cases}w(f) & \text { if } t\left(e_{g}\right)=o\left(f_{h}\right), \quad \tilde{c}\left(e_{g}, f_{h}\right):=\left\{\begin{array}{ll}
1 & \text { if } e_{g}^{-1}=f_{h} \\
0 & \text { otherwise }
\end{array}\right. \text { otherwise }\end{cases}
$$

For $g \in \Gamma$, let the matrix $\mathbf{B}_{g}=\left(b_{e f}^{(g)}\right)$ be defined by

$$
b_{e f}^{(g)}:= \begin{cases}w(f) & \text { if } \alpha(e)=g \text { and } t(e)=o(f) \\ 0 & \text { otherwise. }\end{cases}
$$

Furthermore, let the matrix $\mathbf{J}_{g}=\left(c_{e f}^{(g)}\right)$ be defined by

$$
c_{e f}^{(g)}:= \begin{cases}1 & \text { if } \alpha(e)=g \text { and } e^{-1}=f \\ 0 & \text { otherwise. }\end{cases}
$$

Theorem 5 Let $G$ be a connected graph with $l$ unoriented edges, $\mathbf{W}=\mathbf{W}(G)$ a weighted matrix of $G, \Gamma$ a finite group and $\alpha: D(G) \longrightarrow \Gamma$ an ordinary voltage assignment. Furthermore, let $\rho_{1}=1, \rho_{2}, \cdots, \rho_{k}$ be all inequivalent irreducible representations of $\Gamma$, and $f_{i}$ the degree of $\rho_{i}$ for each $i$, where $f_{1}=1$. Suppose that the $\Gamma$-covering $G^{\alpha}$ of $G$ is connected. Then the reciprocal of the zeta function of $G^{\alpha}$ is

$\zeta_{1}\left(G^{\alpha}, \tilde{w}, u, t\right)^{-1}=\zeta_{1}(G, w, u, t)^{-1} \cdot \prod_{i=2}^{k} \operatorname{det}\left(\mathbf{I}_{2 l f_{i}}-t \sum_{h \in \Gamma} \rho_{i}(h) \bigotimes\left(\mathbf{B}_{h}-(1-u) \mathbf{J}_{h}\right)\right)^{f_{i}}$

Proof. Let $D(G)=\left\{e_{1}, \cdots, e_{l}, e_{l+1}, \cdots, e_{2 l}\right\}$ and $\Gamma=\left\{1=g_{1}, g_{2}, \cdots, g_{m}\right\}$. Arrange arcs of $G^{\alpha}$ in $m$ blocks: $\left(e_{1}, 1\right), \cdots,\left(e_{2 l}, 1\right) ;\left(e_{1}, g_{2}\right), \cdots,\left(e_{2 l}, g_{2}\right) ; \cdots$; $\left(e_{1}, g_{m}\right), \cdots,\left(e_{2 l}, g_{m}\right)$. We consider the matrix $\tilde{\mathbf{B}}-\tilde{\mathbf{J}}_{0}$ under this order. For $h \in \Gamma$, let $\mathbf{P}_{h}=\left(p_{i j}^{(h)}\right)$. Suppose that $p_{i j}^{(h)}=1$, i.e., $g_{j}=g_{i} h$. Then $\left.t\left(e, g_{i}\right)=o\left(f, g_{j}\right)\right)$ if and only if $t(e)=o(f)$ and $\left(o(f), g_{j}\right)=o\left(f, g_{j}\right)=t\left(e, g_{i}\right)=$ $\left(t(e), g_{i} \alpha(e)\right)$, i.e., $\alpha(e)=g_{i}^{-1} g_{j}=g_{i}^{-1} g_{i} h=h$. Thus we have

$$
\tilde{\mathbf{B}}-(1-u) \tilde{\mathbf{J}}_{0}=\sum_{h \in \Gamma} \mathbf{P}_{h} \bigotimes\left(\mathbf{B}_{h}-(1-u) \mathbf{J}_{h}\right)
$$

Let $\rho$ be the right regular representation of $\Gamma$. Furthermore, let $\rho_{1}=$ $1, \rho_{2}, \cdots, \rho_{t}$ be all inequivalent irreducible representations of $\Gamma$, and $f_{i}$ the degree of $\rho_{i}$ for each $i$, where $f_{1}=1$. Then we have $\rho(h)=\mathbf{P}_{h}$ for $h \in \Gamma$. 
Furthermore, there exists a regular matrix $\mathbf{P}$ such that $\mathbf{P}^{-1} \rho(h) \mathbf{P}=(1) \oplus$ $f_{2} \circ \rho_{2}(h) \oplus \cdots \oplus f_{t} \circ \rho_{t}(h)$ for each $h \in \Gamma$. Putting $\mathbf{F}=\left(\mathbf{P}^{-1} \otimes \mathbf{I}_{2 l}\right)(\tilde{\mathbf{B}}-$ $\left.\tilde{\mathbf{J}}_{0}\right)\left(\mathbf{P} \otimes \mathbf{I}_{2 l}\right)$, we have

$$
\mathbf{F}=\sum_{h \in \Gamma}\left\{(1) \oplus f_{2} \circ \rho_{2}(h) \oplus \cdots \oplus f_{t} \circ \rho_{t}(h)\right\} \bigotimes\left(\mathbf{B}_{h}-(1-u) \mathbf{J}_{h}\right) .
$$

Note that $\mathbf{B}-\mathbf{J}_{0}=\sum_{h \in \Gamma}\left(\mathbf{B}_{h}-\mathbf{J}_{h}\right)$ and $1+f_{2}^{2}+\cdots+f_{t}^{2}=m$. Therefore it follows that

$$
\begin{aligned}
\zeta_{1}\left(G^{\alpha}, \tilde{w}, u, t\right)^{-1} & =\operatorname{det}\left(\mathbf{I}_{2 l m}-t\left(\tilde{\mathbf{B}}-(1-u) \tilde{\mathbf{J}}_{0}\right)\right) \\
& =\operatorname{det}\left(\mathbf{I}_{2 l}-t\left(\mathbf{B}-(1-u) \mathbf{J}_{0}\right)\right) \prod_{i=2}^{k} \operatorname{det}\left(\mathbf{I}_{2 l f_{i}}-t \sum_{h} \rho_{i}(h) \otimes\left(\mathbf{B}_{h}-(1-u) \mathbf{J}_{h}\right)\right)^{f_{i}}
\end{aligned}
$$

\section{$4 \quad L$-functions of graphs}

Let $G$ be a connected graph with $n$ vertices and $l$ unoriented edges, $\mathbf{W}=$ $\mathbf{W}(G)$ a weighted matrix of $G, \Gamma$ a finite group and $\alpha: D(G) \longrightarrow \Gamma$ an ordinary voltage assignment. For each path $P=\left(e_{1}, \cdots, e_{r}\right)$ of $G$, set $\alpha(P)=$ $\alpha\left(e_{1}\right) \cdots \alpha\left(e_{r}\right)$. This is called the net voltage of $P$. Furthermore, let $\rho$ be a unitary representation of $\Gamma$ and $d$ its degree. The $L$-function of $G$ associated with $\rho$ and $\alpha$ is defined by

$$
\zeta_{1}(G, w, u, t, \rho, \alpha)=\operatorname{det}\left(\mathbf{I}_{2 l d}-t \sum_{h \in \Gamma} \rho(h) \bigotimes\left(\mathbf{B}_{h}-(1-u) \mathbf{J}_{h}\right)\right)^{-1} .
$$

If $w(e)=1$ for each $e \in D(G)$, then the $L$-function of $G$ is the Bartholdi $L$-function of $G$ :

$$
\zeta_{1}(G, w, u, t, \rho, \alpha)=\zeta_{G}(u, t, \rho, \alpha)=\prod_{[C]} \operatorname{det}\left(\mathbf{I}_{d}-\rho(\alpha(C)) u^{c b c(C)} t^{|C|}\right)^{-1},
$$

where $\operatorname{cbc}(C)$ is the cyclic bump count of $V$, and $[C]$ runs over all equivalence classes of prime, reduced cycles of $G$ (see [8]). In the case of $u=0$, then the $L$-function of $G$ is the new $L$-function of $G$ (see [9]).

A determinant expression for the $L$-function of $G$ associated with $\rho$ and $\alpha$ is given as follows. Let $1 \leq i, j \leq n$. Then, the $(i, j)$-block $\mathbf{F}_{i, j}$ of a $d n \times d n$ matrix $\mathbf{F}$ is the submatrix of $\mathbf{K}$ consisting of $d(i-1)+1, \cdots, d i$ rows and $d(j-1)+1, \cdots, d j$ columns.

Theorem 6 Let $G$ be a connected graph with $n$ vertices and $l$ unoriented edges, $\mathbf{W}(G)$ a weighted matrix of $G, \Gamma$ a finite group and $\alpha: D(G) \longrightarrow \Gamma$ an ordinary voltage assignment. Furthermore, let $\rho$ a representation of $\Gamma$ and $d$ the degree of $\rho$. Then the reciprocal of the L-function of $G$ associated with $\rho$ and $\alpha$ is

$\zeta_{1}(G, w, u, t, \rho, \alpha)^{-1}=\left(1-(1-u)^{2} t^{2}\right)^{(l-n) d} \operatorname{det}\left(\mathbf{I}_{n d}-t \sum_{h \in \Gamma} \rho(h) \bigotimes \mathbf{W}_{h}+(1-u) t^{2}\left(\mathbf{I}_{d} \bigotimes\left(\mathbf{D}-(1-u) \mathbf{I}_{n}\right)\right)\right)$. 
Proof. The argument is an analogue of Bass' method [2].

Let $\rho$ be a unitary representation of $\Gamma$, and $d$ the degree of $\rho$. Furthermore, let $D(G)=\left\{e_{1}, \cdots, e_{l}, e_{l+1}, \cdots, e_{2 l}\right\}$ such that $e_{l+i}=e_{i}^{-1}(1 \leq i \leq l)$. Let $\mathbf{K}=\left(\mathbf{K}_{i, j}\right)_{1 \leq i \leq 2 l ; 1 \leq j \leq n}$ be the $2 l d \times n d$ matrix defined as follows:

$$
\mathbf{K}_{i, j}:= \begin{cases}w\left(e_{i}\right)^{t} \mathbf{I}_{d} & \text { if } o\left(e_{i}\right)=v_{j} \\ \mathbf{0}_{d} & \text { otherwise. }\end{cases}
$$

Furthermore, we define two $2 l d \times n d$ matrices $\mathbf{L}=\left(\mathbf{L}_{i, j}\right)_{1 \leq i \leq 2 l ; 1 \leq j \leq n}$ and $\mathbf{H}=$ $\left(\mathbf{H}_{i, j}\right)_{1 \leq i \leq 2 l ; 1 \leq j \leq n}$ as follows:

$$
\mathbf{L}_{i, j}:=\left\{\begin{array}{ll}
\rho\left(\alpha\left(e_{i}\right)\right) & \text { if } t\left(e_{i}\right)=v_{j}, \\
\mathbf{0}_{d} & \text { otherwise. }
\end{array}, \mathbf{H}_{i, j}:= \begin{cases}\mathbf{I}_{d} & \text { if } o\left(e_{i}\right)=v_{j}, \\
\mathbf{0}_{d} & \text { otherwise }\end{cases}\right.
$$

Then we have

$$
\mathbf{L}^{t} \mathbf{K}=\sum_{h \in \Gamma} \mathbf{B}_{h} \bigotimes \rho(h)
$$

and

$$
{ }^{t} \mathbf{K L}=\sum_{g \in \Gamma} \mathbf{W}_{g} \bigotimes \rho(g)
$$

Furthermore,

$$
{ }^{t} \mathbf{H K}=\mathbf{D}_{d}=\mathbf{D} \bigotimes \mathbf{I}_{d},
$$

where ${ }^{t} \mathbf{F}$ is the transpose of $\mathbf{F}$.

Now, let

$\mathbf{M}=\left[\begin{array}{cc}\mathbf{0} & w\left(e_{1}^{-1}\right) \rho\left(\alpha\left(e_{1}\right)\right) \oplus \cdots \oplus w\left(e_{l}^{-1}\right) \rho\left(\alpha\left(e_{l}\right)\right) \\ w\left(e_{1}\right) \rho\left(\alpha\left(e_{1}\right)\right)^{-1} \oplus \cdots \oplus w\left(e_{l}\right) \rho\left(\alpha\left(e_{l}\right)\right)^{-1} & \mathbf{0}\end{array}\right]$ and

$$
\mathbf{N}=\mathbf{B}_{\rho}-\mathbf{M}
$$

where $\mathbf{B}_{\rho}=\sum_{h \in \Gamma} \mathbf{B}_{h} \otimes \rho(h)$. Then we have

$$
\mathbf{K}^{t} \mathbf{H}={ }^{t} \mathbf{N}^{t} \mathbf{J}_{\rho}+\left(w\left(e_{1}\right) \mathbf{I}_{d} \oplus \cdots \oplus w\left(e_{2 l}\right) \mathbf{I}_{d}\right),
$$

where $\mathbf{J}_{\rho}=\sum_{h \in \Gamma} \mathbf{J}_{h} \otimes \rho(h)$.

We introduce two $(2 l+n) d \times(2 l+n) d$ matrices as follows:

$\mathbf{P}=\left[\begin{array}{cc}\left(1-(1-u)^{2} t^{2}\right) \mathbf{I}_{n d} & -{ }^{t} \mathbf{L}+(1-u) t{ }^{t} \mathbf{H} \\ \mathbf{0} & \mathbf{I}_{2 l d}\end{array}\right], \mathbf{Q}=\left[\begin{array}{cc}\mathbf{I}_{n d} & { }^{t} \mathbf{L}-(1-u) t{ }^{t} \mathbf{H} \\ t \mathbf{K} & \left(1-(1-u)^{2} t^{2}\right) \mathbf{I}_{2 l d}\end{array}\right]$

By (2) and (3), we have

$$
\begin{aligned}
\mathbf{P Q} & =\left[\begin{array}{cc}
\left(1-(1-u)^{2} t^{2}\right) \mathbf{I}_{n d}-t^{t} \mathbf{L K}+(1-u) t^{2}{ }^{t} \mathbf{H K} & \mathbf{0} \\
t \mathbf{K} & \left(1-(1-u)^{2} t^{2}\right) \mathbf{I}_{2 l d}
\end{array}\right] \\
& =\left[\begin{array}{cc}
\left(1-(1-u)^{2} t^{2}\right) \mathbf{I}_{n d}-t \sum_{g \in \Gamma}{ }_{t \mathbf{K}} \mathbf{W}_{g} \otimes^{t} \rho(g)+(1-u) t^{2} \mathbf{D}_{d} & \mathbf{0} \\
&
\end{array}\right]
\end{aligned}
$$


By (1) and (4),

$\mathbf{Q P}=\left[\begin{array}{cc}\left(1-(1-u)^{2} t^{2}\right) \mathbf{I}_{n d} & \mathbf{0} \\ t\left(1-(1-u)^{2} t^{2}\right) \mathbf{K} & -t \mathbf{K}^{t} \mathbf{L}+(1-u) t^{2} \mathbf{K}^{t} \mathbf{H}+\left(1-(1-u)^{2} t^{2}\right) \mathbf{I}_{2 l d}\end{array}\right]$.

Since

$$
w\left(e_{1}\right) \mathbf{I}_{d} \oplus \cdots \oplus w\left(e_{2 l}\right) \mathbf{I}_{d}={ }^{t} \mathbf{M}^{t} \mathbf{J}_{\rho}
$$

and $\left({ }^{t} \mathbf{J}_{\rho}\right)^{2}=\mathbf{I}_{2 l d}$, we have

$$
\begin{aligned}
& -t \mathbf{K}^{t} \mathbf{L}+(1-u) t^{2} \mathbf{K}^{t} \mathbf{H}+\left(1-(1-u)^{2} t^{2}\right) \mathbf{I}_{2 l d} \\
= & \mathbf{I}_{2 l d}-t\left({ }^{t} \mathbf{N}+{ }^{t} \mathbf{M}\right)+(1-u) t^{2}\left({ }^{t} \mathbf{N}^{t} \mathbf{J}_{\rho}+{ }^{t} \mathbf{M}^{t} \mathbf{J}_{\rho}-(1-u){ }^{t} \mathbf{J}_{\rho}{ }^{t} \mathbf{J}_{\rho}\right) \\
= & \left(\mathbf{I}_{2 l d}-t\left({ }^{t} \mathbf{N}+{ }^{t} \mathbf{M}-(1-u){ }^{t} \mathbf{J}_{\rho}\right)\right)\left(\mathbf{I}_{2 l d}-(1-u) t^{t} \mathbf{J}_{\rho}\right) .
\end{aligned}
$$

Thus,

$\mathbf{Q P}=\left[\begin{array}{cc}\left(1-(1-u)^{2} t^{2}\right) \mathbf{I}_{n d} & \mathbf{0} \\ t\left(1-(1-u)^{2} t^{2}\right) \mathbf{K} & \left(\mathbf{I}_{2 l d}-t\left({ }^{t} \mathbf{N}+{ }^{t} \mathbf{M}-(1-u)^{t} \mathbf{J}_{\rho}\right)\right)\left(\mathbf{I}_{2 l d}-(1-u) t^{t} \mathbf{J}_{\rho}\right)\end{array}\right]$.

Since $\operatorname{det}(\mathbf{P Q})=\operatorname{det}(\mathbf{Q P})$, we have

$$
\begin{aligned}
& \left(1-(1-u)^{2} t^{2}\right)^{2 l d} \operatorname{det}\left(\mathbf{I}_{n d}-t \sum_{g \in \Gamma}{ }^{t} \mathbf{W}_{g} \bigotimes^{t} \rho(g)+(1-u)\left(\mathbf{D}_{d}-(1-u) \mathbf{I}_{n d}\right) t^{2}\right) \\
& =\left(1-(1-u)^{2} t^{2}\right)^{n d} \operatorname{det}\left(\mathbf{I}_{2 l d}-t\left({ }^{t} \mathbf{N}+{ }^{t} \mathbf{M}-(1-u)^{t} \mathbf{J}_{\rho}\right)\right) \operatorname{det}\left(\mathbf{I}_{2 l d}-(1-u) t^{t} \mathbf{J}_{\rho}\right) .
\end{aligned}
$$

But,

$$
\begin{aligned}
\operatorname{det}\left(\mathbf{I}_{2 l d}-(1-u) t^{t} \mathbf{J}_{\rho}\right) & =\operatorname{det}\left(\left[\begin{array}{cc}
\mathbf{I}_{l d} & (1-u) t \mathbf{S} \\
\mathbf{0} & \mathbf{I}_{l d}
\end{array}\right]\right) \operatorname{det}\left(\left[\begin{array}{cc}
\mathbf{I}_{l d} & -(1-u) t \mathbf{S} \\
-(1-u) t \mathbf{S}^{-1} & \mathbf{I}_{l d}
\end{array}\right]\right) \\
& =\operatorname{det}\left(\left[\begin{array}{cc}
\left(1-(1-u)^{2} t^{2}\right) \mathbf{I}_{l d} & \mathbf{0} \\
* & \mathbf{I}_{l d}
\end{array}\right]\right)=\left(1-(1-u)^{2} t^{2}\right)^{l d},
\end{aligned}
$$

where $\mathbf{S}=\rho\left(\alpha\left(e_{1}\right)\right) \oplus \cdots \oplus \rho\left(\alpha\left(e_{l}\right)\right)$. Therefore it follows that

$$
\begin{gathered}
\left(1-(1-u)^{2} t^{2}\right)^{2 l d} \operatorname{det}\left(\mathbf{I}_{n d}-t \sum_{g \in \Gamma}{ }^{t} \mathbf{W}_{g} \bigotimes^{t} \rho(g)+(1-u)\left(\mathbf{D}_{d}-(1-u) \mathbf{I}_{n d}\right) t^{2}\right) \\
=\left(1-(1-u)^{2} t^{2}\right)^{(l+n) d} \operatorname{det}\left(\mathbf{I}_{2 l d}-t\left({ }^{t} \mathbf{N}+{ }^{t} \mathbf{M}-(1-u)^{t} \mathbf{J}_{\rho}\right)\right) .
\end{gathered}
$$

Hence

$$
\begin{aligned}
& \left.\operatorname{det}\left(\mathbf{I}_{2 l d}-t \sum_{h} \rho(h) \otimes\left(\mathbf{B}_{h}-(1-u) \mathbf{J}_{h}\right)\right)\right) \\
= & \left(1-(1-u)^{2} t^{2}\right)^{(l-n) d} \operatorname{det}\left(\mathbf{I}_{n d}-t \sum_{g \in \Gamma} \rho(g) \otimes \mathbf{W}_{g}+(1-u)\left(\mathbf{I}_{d} \otimes\left(\mathbf{D}-(1-u) \mathbf{I}_{n}\right)\right) t^{2}\right) .
\end{aligned}
$$

By Theorems 4,5,6, the following result holds. 
Corollary 1 Let $G$ be a connected graph, $\mathbf{W}(G)$ a weighted matrix of $G, \Gamma$ a finite group and $\alpha: D(G) \longrightarrow \Gamma$ an ordinary voltage assignment. Then we have

$$
\zeta_{1}\left(G^{\alpha}, \tilde{w}, u, t\right)=\prod_{\rho} \zeta_{1}(G, w, u, t, \rho, \alpha)^{\operatorname{deg} \rho},
$$

where $\rho$ runs over all inequivalent irreducible representations of $\Gamma$.

In the case that $w\left(v_{i}, v_{j}\right)=1$ for each $\left(v_{i}, v_{j}\right) \in D(G)$, we obtain a decomposition formula for the Bartholdi zeta function of a regular covering of a graph by Mizuno and Sato [8].

Corollary 2 (Mizuno and Sato) Let $G$ be a connected graph, $\Gamma$ a finite group and $\alpha: D(G) \longrightarrow \Gamma$ an ordinary voltage assignment. Suppose that the $\Gamma$-covering $G^{\alpha}$ of $G$ is connected. Then we have

$$
\zeta\left(G^{\alpha}, u, t\right)=\prod_{\rho} \zeta_{G}(u, t, \rho, \alpha)^{\operatorname{deg} \rho},
$$

where $\rho$ runs over all inequivalent irreducible representations of $\Gamma$.

If $u=0$, we obtain a decomposition formula for the new zeta function of a regular covering of a graph by Sato [9].

Corollary 3 (Sato) Let $G$ be a connected graph, $\mathbf{W}(G)$ a weighted matrix of $G, \Gamma$ a finite group and $\alpha: D(G) \longrightarrow \Gamma$ an ordinary voltage assignment. Then we have

$$
\mathbf{Z}_{1}\left(G^{\alpha}, \tilde{w}, t\right)=\prod_{\rho} \mathbf{Z}_{1}(G, w, t, \rho, \alpha)^{\operatorname{deg} \rho},
$$

where $\rho$ runs over all inequivalent irreducible representations of $\Gamma$.

\section{Example}

Finally, we give an example. Let $G=K_{3}$ be the complete graph with three vertices $v_{1}, v_{2}, v_{3}$, and let the following matrix $\mathbf{W}$ be a weighted matrix of $K_{3}$ :

$$
\mathbf{W}=\left[\begin{array}{lll}
0 & a & b \\
p & 0 & c \\
q & r & 0
\end{array}\right]
$$

Then, by Theorem 3, we have

$$
\begin{gathered}
\zeta_{1}\left(K_{3}, w, u, t\right)^{-1}=\operatorname{det}\left(\mathbf{I}_{3}-t \mathbf{W}+(1-u) t^{2}\left(\mathbf{D}-(1-u) \mathbf{I}_{3}\right)\right) \\
\operatorname{det}\left(\left[\begin{array}{ccc}
1+(1-u)(a+b-1+u) t^{2} & -t a & -t b \\
-t p & 1+(1-u)(p+c-1+u) t^{2} & -t c \\
-t q & -t r & 1+(1-u)(q+r-1+u) t^{2}
\end{array}\right]\right)
\end{gathered}
$$




$$
=\alpha \beta \gamma-(b q \beta+a p \gamma+c r \alpha) t^{2}-(a c q+b p r) t^{3},
$$

where $\alpha=1+(1-u)(a+b-1+u) t^{2}, \beta=1+(1-u)(p+c-1+u) t^{2}$ and $\gamma=1+(1-u)(q+r-1+u) t^{2}$.

Let $\Gamma=Z_{3}=\left\{1, \tau, \tau^{2}\right\}\left(\tau^{3}=1\right)$ be the cyclic group of order 3 , and let $\alpha: D\left(K_{3}\right) \longrightarrow Z_{3}$ be the ordinary voltage assignment such that $\alpha\left(v_{1}, v_{2}\right)=\tau$ and $\alpha\left(v_{1}, v_{3}\right)=\alpha\left(v_{2}, v_{3}\right)=1$. Then, the $\mathbf{Z}_{3}$-covering $K_{3}{ }^{\alpha}$ is the cycle graph $C_{9}$ with nine vertices. The characters of $\mathbf{Z}_{3}$ are given as follows: $\chi_{i}\left(\tau^{j}\right)=\left(\xi^{i}\right)^{j}$, $0 \leq i, j \leq 2$, where $\xi=\frac{-1+\sqrt{-3}}{2}$.

Now, we present the $L$-function $\mathbf{Z}_{1}\left(K_{3}, w, u, \chi_{1}, \alpha\right)$ of $K_{3}$ associated with $\chi_{1}$ and $\alpha$. Theorem 6 implies that

$$
\begin{aligned}
\zeta_{1}\left(K_{3}, w, u, t, \chi_{1}, \alpha\right)^{-1} & =\operatorname{det}\left(\mathbf{I}_{3}-t \sum_{i=0}^{2} \chi_{1}\left(\tau^{i}\right) \mathbf{W}_{\tau^{i}}+(1-u) t^{2}\left(\mathbf{D}-(1-u) \mathbf{I}_{3}\right)\right) \\
& =\operatorname{det}\left(\left[\begin{array}{ccc}
\alpha & -\xi t a & -t b \\
-\xi^{2} t p & \beta & -t c \\
-t q & -t r & \gamma
\end{array}\right]\right) \\
& =\alpha \beta \gamma-(b q \beta+a p \gamma+c r \alpha) t^{2}-\left(a c q \xi+b p r \xi^{2}\right) t^{3} .
\end{aligned}
$$

Similarly, we have

$$
\zeta_{1}\left(K_{3}, w, u, t, \chi_{2}, \alpha\right)^{-1}=\alpha \beta \gamma-(b q \beta+a p \gamma+c r \alpha) t^{2}-\left(a c q \xi^{2}+b p r \xi\right) t^{3} .
$$

By Corollary 1, it follows that

$$
\begin{gathered}
\zeta_{1}\left(C_{9}, w, u, t\right)^{-1}=\zeta_{1}\left(K_{3}^{\alpha}, w, u, t\right)^{-1}=\zeta_{1}\left(K_{3}, w, u, t\right)^{-1} \zeta_{1}\left(K_{3}, w, u, t, \chi_{1}, \alpha\right)^{-1} \zeta_{1}\left(K_{3}, w, u, t, \chi_{2}, \alpha\right)^{-1} \\
=\left\{\alpha \beta \gamma-(b q \beta+a p \gamma+c r \alpha) t^{2}-(a c q+b p r) t^{3}\right\}\left\{\left(\alpha \beta \gamma-(b q \beta+a p \gamma+c r \alpha) t^{2}\right)^{2}\right. \\
\left.+(a c q+b p r)\left(\alpha \beta \gamma-(b q \beta+a p \gamma+c r \alpha) t^{2}\right) t^{3}+\left(a^{2} c^{2} q^{2}-a b c p q r+b^{2} p^{2} r^{2}\right) t^{6}\right\} .
\end{gathered}
$$

\section{References}

[1] L. Bartholdi, Counting paths in graphs, Enseign. Math. 45 (1999), 83-131.

[2] H. Bass, The Ihara-Selberg zeta function of a tree lattice, Internat. J. Math. 3 (1992), 717-797.

[3] D. Foata and D. Zeilberger, A combinatorial proof of Bass's evaluations of the Ihara-Selberg zeta function for graphs, Trans. Amer. Math. Soc. 351 (1999), 2257-2274.

[4] J. L. Gross and T. W. Tucker, Topological Graph Theory, WileyInterscience, New York, 1987. 
[5] K. Hashimoto, Zeta Functions of Finite Graphs and Representations of $p$ Adic Groups, in "Adv. Stud. Pure Math". Vol. 15, pp. 211-280, Academic Press, New York, 1989.

[6] Y. Ihara, On discrete subgroups of the two by two projective linear group over p-adic fields, J. Math. Soc. Japan 18 (1966), 219-235.

[7] M. Kotani and T. Sunada, Zeta functions of finite graphs, J. Math. Sci. U. Tokyo 7 (2000), 7-25.

[8] H. Mizuno and I. Sato, Bartholdi zeta functions of graph coverings, $J$. Combin. Theory Ser. B 89 (2003), 27-41.

[9] I. Sato, A new zeta function of a graph, preprint.

[10] J. -P. Serre, Linear Representations of Finite Group, Springer-Verlag, New York, 1977.

[11] H. M. Stark and A. A. Terras, Zeta functions of finite graphs and coverings, Adv. Math. 121 (1996), 124-165.

[12] T. Sunada, L-Functions in Geometry and Some Applications, in "Lecture Notes in Math"., Vol. 1201, pp. 266-284, Springer-Verlag, New York, 1986.

[13] T. Sunada, Fundamental Groups and Laplacians(in Japanese), Kinokuniya, Tokyo, 1988.

\section{Received: November 17, 2006}

